Background Current international guidelines on HIV Pre-Exposure Prophylaxis (PrEP) recommend serological screening for syphilis at routine three-monthly PrEP appointments. The aim of our study was to describe the pattern of clinical presentation of syphilis among men who have sex with men (MSM) taking PrEP. We were interested in whether syphilis is detected through screening at scheduled three-monthly PrEP clinic appointments or whether primary or secondary syphilis presented at unscheduled interval visits.

Methods This was a retrospective study of MSM attending the PrEP clinic at the Melbourne Sexual Health Centre between February 2016 and March 2019. Serological screening for syphilis was routinely undertaken at three-monthly PrEP clinic appointments. Diagnoses of early syphilis were identified from PrEP clinic visits and from interim walk-in STI clinic attendances.

Results There were 69 cases of early syphilis among 61 MSM taking PrEP during the study period. There were 24 (35\%) primary, 16 (23\%) secondary and 29 (42\%) early latent infections. The incidence of early syphilis was 8.6 per 100 personyears. A substantial proportion of primary (58\%) and secondary (44\%) syphilis diagnoses were made at interim STI clinic attendances, between PrEP appointments.

Conclusions Syphilis screening at routine three-monthly PrEP visits alone fails to detect a proportion of primary and secondary syphilis infections and may be insufficient in preventing onward transmission. Education of MSM taking PrEP regarding the risk of syphilis and symptom recognition is necessary together with access to syphilis testing between PrEP visits.

\section{P157 WOMEN COMMONLY USE SEXUAL ENRICHMENT AIDS AND RARELY RECEIVE INFORMATION FROM MEDICAL PRACTITIONERS ON HYGIENE AND SAFETY}

A Collar*, S Core, K Frietze. University of New Mexico, Albuquerque, USA

10.1136/sextrans-2021-sti.262

Sexual enrichment aids (i.e. sex toys) are devices used to enhance the sexual experience of individuals. However, our hypothesis is that transmission of STIs could also occur via fomites, like sexual enrichment aids (SEAs, i.e. vaginally insertable sex toys). The aim of this research is to understand women's sexual behaviors associated with SEA use.

Methods Utilizing mix methodology, we investigated the prevalence of SEA use among women, evaluated behaviors associated with SEA use, and examined if medical practitioners have counseled women on safe SEA use. We recruited 800 sexually active women between the ages of 18-35 years old to participate in a cross-sectional study. These women were also invited to participate in a qualitative interview to further explore their sexual behaviors.

Results We found that SEA use is common, with $79.8 \%$ of all women reporting SEA use. When women were stratified based on sexual orientation, we found that SEA use is even more common among WSW and WSWM, at $89.7 \%$ and $92 \%$, respectively. We also found that at least of subset of women engage in behaviors that may transmit infection, including sharing SEAs with partners, infrequent barrier protection use, and inconsistent cleaning practices. Additionally, the majority of women are not counseled on SEA use and hygiene by medical practitioners. Among women who report having received counsel from medical practitioners on SEA use and hygiene, WSW and WSWM are not counseled as extensively as their WSM counterparts.

Conclusions We found that SEA use is common among women and that at least a subset of women engage in behaviors that may have the potential to transmit infection. This study provides insight to a common sexual behavior among women and points to the need for more studies to provide evidence-based medical counseling to women.

\section{P161 ANAL CANCER SCREENING PRACTICES: CURRENT EXPERT OPINIONS}

${ }^{1} \mathrm{R}$ Plotzker* ${ }^{*}{ }^{2} \mathrm{G}$ Barnell, ${ }^{3} \mathrm{D}$ Wiley, ${ }^{4} \mathrm{E}$ Stier, ${ }^{5} \mathrm{~N}$ Jay. ${ }^{1}$ Department of Epidemiology and Biostatistics, University of California, San Francisco, San Francisco, USA; ${ }^{2}$ Department of Surgery, Oakland Medical Center, Kaiser Permanente Northern California, Oakland, USA; ${ }^{3}$ University of California Los Angeles School of Nursing, LOS Angeles, USA; ${ }^{4}$ Boston University School of Medicine, Boston, USA; ${ }^{5}$ University of California, San Francisco, San Francisco, USA

\subsection{6/sextrans-2021-sti.263}

Background Anal cancer incidence is high among people with HIV (PWH), men who have sex with men (MSM), and women with lower genital tract disease (LGTD). Anal cancer is preceded by persistent high-risk human papillomavirus infection and subsequent high-grade squamous intraepithelial lesions (HSIL). Emerging screening practices may reduce anal cancer incidence. However, no national or international screening guidance exists. The International Anal Neoplasia Society (IANS) solicited expert opinion to understand preferred current anal screening practices.

Methods IANS members and meeting attendees were invited to complete an online survey of screening practices. Screening initiation age, screening tool, and high resolution anoscopy (HRA) referral threshold were assessed. Recommendations for age and tool were delineated for PWH, sub-grouped female/ non-MSM-male/MSM; and for women with LGTD, subgrouped by disease (HSIL/cancer) and anatomic site (vulvar/ cervico-vaginal). HRA referral threshold compared immunocompetent versus immunocompromised patients.

Results Of 1150 individuals contacted, 140 (12\%) participated and were included; 113 (81\%) were HRA providers. Similar proportions of respondents specified a screening initiation age in all PWH (range 61-64\%); however, there was no consensus for the specific age to begin screening for any PWH sub-group. More respondents preferred 'no age restriction' for MSM (36\%) compared to non-MSM-male counterparts (22\%). Among LGTD groups, 'no age restriction' was more commonly preferred (range 44-55\%). Of tools, across all PWH and LGTD groups, cytology (79-89\%) and digital anorectal exam (DARE) (73-83\%) were most frequently recommended. For HRA referral threshold, 'any abnormality' was more often selected for both immunocompromised (56\%) and immunocompetent (46\%) patients than a specific cytology result $(29 \%, 36 \%)$. Among those who specified cytology, consensus was lacking and did not vary by immune status.

Conclusion Respondents concurred cytology and DARE were preferred screening modalities. Screening initiation age and HRA referral threshold showed less consensus. Evidence-based guidance is needed and may lead to more consistent screening practices. 